\title{
Could vagus nerve stimulation have a role in the treatment of diabetes?
}

\author{
Charles-Henri Malbert ${ }^{*}, 1$ \\ ${ }^{1}$ Département de Nutrition Humaine, INRA, 35590 Saint-Gilles, France \\ * Author for correspondence: Tel.: +33 1 (0)2 234850 71; Fax: +33 1 (0)2 234850 80; charles-henri.malbert@inra.fr
}

\begin{abstract}
“Traditional bariatric surgery is primarily conceived as an intervention that reduces the risk of future disease (i.e., to prevent metabolic or cardiovascular complications of severe obesity) rather than as an approach to treat established disease. On the contrary, metabolic surgery should be considered as a means to achieve the glycemic control necessary to reduce the risk of microvascular complications and cardiovascular disease."
\end{abstract}

First draft submitted: 28 August 2017; Accepted for publication: 25 September 2017; Published online: 3 November 2017

Type 2 diabetes (T2D) is characterized by relative insulin deficiency caused by pancreatic $\beta$-cell dysfunction and insulin resistance in target organs: liver, skeletal muscle, kidneys, brain, intestine and adipose tissue. A total of 171 million individuals were estimated to have diabetes in the year 2000, and this is expected to increase to 366 million by 2030 [1]. T2D was the sixth leading cause of disability in 2015 as a consequence of the morbidity and mortality associated with macro- and micro-vascular complications including cardiovascular disease and stroke, retinopathy, nephropathy and neuropathy. Obesity, physical inactivity and energy-dense diets are common factors that occur in synchrony leading to the pathogenesis of T2D, together with a specific phenotype that appears to increase the risk of cardiovascular death and other life-threatening diseases. However, obesity itself appears an essential contributor since $60 \%$ of patients with T2D are obese (body mass index $\geq 30 \mathrm{~kg} / \mathrm{m}^{2}$ ) [2].

Glucose control remains a major focus in the management of patients with T2D. Indeed, while there is a consensus demonstrating that reducing hyperglycemia decreases the onset and progression of microvascular complications, the impact of glucose control on cardiovascular complications is more modest yet likely to be present after many years of improved control. Accordingly, personalized medicine is necessary, balancing the benefits of glycemic control with its potential risks, taking into account the adverse effects of glucose-lowering medications (e.g., particularly hypoglycemia) and the patient's age and health status. Recently our group has been focusing on vagal stimulation as a minimally invasive strategy for weight loss and improvement of insulin sensitivity [3-6]. Together with new surgical approaches and innovative, stimulating schemes, this might provide an interesting alternative therapeutic approach. To put vagal stimulation in perspective, we will present the actual consensus for treatment of T2D that includes pharmacological and surgical glycemic control together with the emerging technologies that, at present, have limitations in sample size and relatively short-term follow-up. We will discuss the potential place of vagal stimulation in the decision algorithm with specific reference to the probable side effects. At last, the future developments will be outlined.

Until recently, there was no clear consensus on a therapeutic algorithm for T2D, that includes both metabolic surgery and pharmacological/lifestyle interventions. In 2016, a joint statement by international diabetes organizations refined the treatment algorithm to insert metabolic surgery since clinical evidence from numerous randomized clinical trials support the inclusion of metabolic surgery among antidiabetes interventions [7]. The availability of several molecular groups acting on most of the key glucose metabolic pathways has profoundly improved the management of hyperglycemia. While insulin therapy is the most effective treatment regarding overall glycemic control, it is associated with increased risk of hypoglycemia. Therefore, it is considered only after several months of unsuccessful glycemic control. Metformin alone, or later as dual or triple association with sulfonylurea, thiazolidinediones, DPP4 inhibitors, SGLT2 inhibitors or GLP1 receptor agonists, are part of antihyperglycemic therapy in T2D [8]. New concerns about DDP-4 inhibitors and heart failure and the issues concerning SGLT2 inhibitors and renal status [9] on the one hand and excellent glycemic control together with the reduction of cardiovascular risk 
factors obtained with metabolic surgery, on the other, suggest that the impact of the later therapeutic option will increase in the future. Because of the irreversibility of metabolic surgery and the potential side effects of several molecular classes of antidiabetic drugs, there has recently been increased interest in device-based gastrointestinal interventions designed to reproduce some of the benefits of metabolic surgery. Small human studies have examined numerous approaches, including space-occupying endoluminal devices, gastric electrical stimulation, duodenal and gastroduodenal endoluminal barriers, and duodenal mucosal resurfacing [10]. Preliminary short-term results show variable degrees of efficacy, depending on the device, in improving glycemic and metabolic control in patients with obesity and T2D.

Using a large animal model of insulin resistance without constant hyperglycemia (e.g., in a prediabetic condition), we found that vagal stimulation induces an increase in whole body glucose uptake that leads to improved insulin sensitivity which itself resulted in a reduction in fasting glucose [5]. We further demonstrated that insulin-mediated hepatic and skeletal muscle glucose uptakes were impaired by obesity and reversed by vagal stimulation. Given the large volume of muscle mass, the restoration of glucose metabolism in the muscle is probably the major quantitative contributor to whole body glucose uptake. Surprisingly, the reduction of weight gain is much less than the recovery of insulin sensitivity. While the restoration of whole body insulin sensitivity was almost complete, the reduction in body weight was about $25 \%$. Similar changes were observed for total fat mass but not visceral fat, which was not altered by vagal stimulation.

While bilateral abdominal vagal stimulation was able to restore insulin sensitivity, unilateral cervical vagal stimulation has minimal effect on body weight and does not affect fasting glycemia. Therefore, the technical challenge of inserting two electrodes around the abdominal vagal trunks needs to be weighed against the positive impact on insulin sensitivity. However, the laparoscopic surgical approach to insert dedicated electrodes on the dorsal and ventral vagus trunks is established, even for centimeter long cuffs. Recently, vagal blockade by high-frequency high-amplitude biphasic pulses on both abdominal vagal trunks for 12 months has been reported to reduce $\mathrm{HbA}_{1 \mathrm{c}}$ by $1 \%$. Nevertheless, a previous study from the same group using the same device failed to demonstrate any weight loss [11]. Results from this study, from the metabolic surgery outcomes, and from our work suggest that a substantial weight loss is not mandatory to improve insulin sensitivity. Equally important to the striking improvement in insulin sensitivity is the absence of autonomic heart balance induced by bilateral vagal stimulation, suggesting that long-term deleterious effects on the heart could be limited. Similarly, we also previously demonstrated that neither gastric emptying of solids or liquids was altered by chronic vagal stimulation in nonobese animals. This suggests that, first, the effect observed on the insulin sensitivity is not related to an altered supply of glucose or glucose-inducting substrates to the small intestine and, second, that epigastric fullness and other elevated gastric retention time symptoms are not likely to occur during vagal stimulation.

Bilateral chronic vagal stimulation with stimulation parameters similar to those used either in epileptic or depressive patients has the potential for glycemic control and weight control. However, at the abdominal or thoracic level, most neurons are small diameter myelinated and unmyelinated neurons, in other words, Ад- or C-type. Therefore, large current pulses are required for depolarizing the axon membrane and thus generating an action potential. We recently ascertained the capability of very high-frequency stimulation schemes (pulsons) to trigger action potentials in abdominal vagal neurons and compared this stimulation scheme on brain metabolism and food intake behavior in conscious pigs [4]. Pulsons activated the brainstem and higher-order brain areas rapidly and consistently after only a couple of days whereas the same was hardly achievable after weeks, using the more classical stimulation scheme. Therefore, it is entirely possible that the improvement in insulin sensitivity observed with $\mathrm{mA}$ amplitude stimulation could be further increased by innovative stimulation systems. Long term evaluation of such stimulation in obese animal models is worth considering.

Traditional bariatric surgery is primarily conceived as an intervention that reduces the risk of future disease (i.e., to prevent metabolic or cardiovascular complications of severe obesity) rather than as an approach to treat established disease. On the contrary, metabolic surgery should be considered as a means to achieve the glycemic control necessary to reduce the risk of microvascular complications and cardiovascular disease. To date, no data have directly demonstrated reductions in such events, compared with standard therapy. Chronic bilateral vagal stimulation also falls within the frame of traditional metabolic surgery: it is obviously extremely successful in the restoration of insulin sensitivity, but long-term efficacy on primary outcomes is still required. At last, a further challenge is translating favorable trial outcomes to a real-world setting and ensuring that evidence-based guidelines are followed in clinical practice. 


\section{References}

1 Wild S, Roglic G, Green A, Sicree R, King H. Global prevalence of diabetes: estimates for the year 2000 and projections for 2030. Diabetes Care 27(5), 1047-1053 (2004).

2 Chatterjee S, Khunti K, Davies MJ. Type 2 diabetes. The Lancet 389(10085), 2239-2251 (2017).

3 Malbert CH, Divoux JL, Maciejasz P, Picq C, Andreu D, Guiraud D. Stimulation of small diameter myelinated and unmyelinated abdominal vagal neurons using high frequency pulses. 7th International IEEE/EMBS Conference on Neural Engineering, Montpellier, France, 510-513 (2015).

4 Malbert C-H, Bobillier E, Picq C, Divoux J-L, Guiraud D, Henry C. Effects of chronic abdominal vagal stimulation of small-diameter neurons on brain metabolism and food intake. Brain Stimulation 10(4), 735-743 (2017).

5 Malbert C-H, Picq C, Divoux J-L, Henry C, Horowitz M. Obesity-associated alterations in glucose metabolism are reversed by chronic bilateral stimulation of the abdominal vagus nerve. Diabetes 66(4), 848-857 (2017).

6 Val-Laillet D, Biraben A, Randuineau G, Malbert CH. Chronic vagus nerve stimulation decreased weight gain, food consumption and sweet craving in adult obese minipigs. Appetite 55(2), 245-252 (2010).

7 Rubino F, Nathan DM, Eckel RH et al. Metabolic surgery in the treatment algorithm for Type 2 diabetes: a joint statement by international diabetes organizations. Diabetes Care 39(6), 861-877 (2016).

8 Inzucchi SE, Bergenstal RM, Buse JB et al. Management of hyperglycemia in Type 2 diabetes, 2015: a patient-centered approach: update to a position statement of the American Diabetes Association and the European Association for the Study of Diabetes. Diabetes Care 38(1), 140-149 (2015).

9 Scirica BM, Braunwald E, Raz I et al. Heart failure, saxagliptin and diabetes mellitus: observations from the SAVOR-TIMI 53 randomized trial. Circulation 130(18), 1579-1588 (2014).

10 Sullivan S, Edmundowicz SA, Thompson CC. Endoscopic bariatric and metabolic therapies: new and emerging technologies. Gastroenterology 152(7), 1791-1801 (2017).

11 The EMPOWER Study Group, Sarr MG, Billington CJ et al. The EMPOWER study: randomized, prospective, double-blind, multicenter trial of vagal blockade to induce weight loss in morbid obesity. Obes Surg. 22(11), 1771-1782 (2012). 